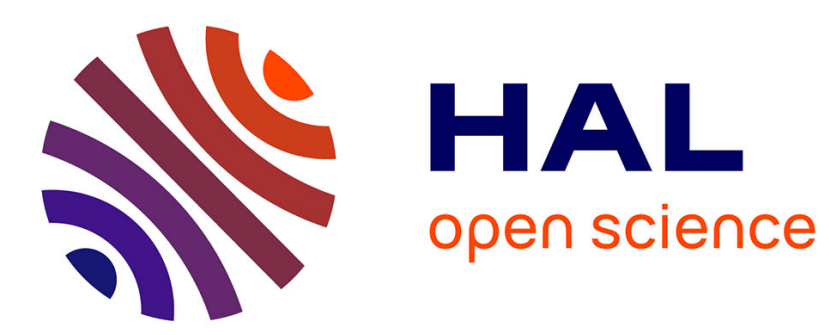

\title{
Stokes flow in a junction of two-dimensional orthogonal channels
}

Mustapha Hellou, Thi Diep Phuong Bach

\section{To cite this version:}

Mustapha Hellou, Thi Diep Phuong Bach. Stokes flow in a junction of two-dimensional orthogonal channels. Zeitschrift für Angewandte Mathematik und Physik, 2011, 62, pp.135-147. 10.1007/s00033010-0087-y · hal-00982491

\section{HAL Id: hal-00982491 \\ https://hal.science/hal-00982491}

Submitted on 23 Apr 2014

HAL is a multi-disciplinary open access archive for the deposit and dissemination of scientific research documents, whether they are published or not. The documents may come from teaching and research institutions in France or abroad, or from public or private research centers.
L'archive ouverte pluridisciplinaire HAL, est destinée au dépôt et à la diffusion de documents scientifiques de niveau recherche, publiés ou non, émanant des établissements d'enseignement et de recherche français ou étrangers, des laboratoires publics ou privés. 


\title{
Stokes Flow in a Junction of two-dimensional Orthogonal Channels
}

\author{
Mustapha HELLOU, Thi Diep Phuong BACH \\ Université Européenne de Bretagne, France \\ INSA, LGCGM, EA3913, F-35708 RENNES
}

Abstract. The purpose of this study is to calculate Stokes flow structures in relation to flow rate distribution in a junction of four orthogonal channels. Particular attention was paid to the existence of recirculation flow inside the channels for low Reynolds numbers. The presence of this recirculation flow may be observed, but only when the flow rate is relatively low in at least one of the branches.

Keywords. Channels; Junction; Flow branches; Stokes eddies; Recirculation flow; Low Reynolds number; Microflows

\section{Introduction}

The problem of viscous flow in a junction of flow branches is encountered in many fundamental and applied situations. Therefore, an understanding of the multiscale transport mechanisms at work at different stages in channel networks is of fundamental interest. Dispersion phenomena in a fracture network is a large-scale example for which the impact of the junction is considered to be a heterogeneity. [Adler and Brenner (1984); Adler and Thovert (1999); Berkowitz et al. (1994); Bruderer and Barnabé (2001); Bryden and Brenner (1996); Dentz and Berkowitz (2003); Mourzenko et al. (2002); Park et al. (1999, 2001, 2003)]. On a microscopic scale, the junction is continuous but microhydrodynamic effects can cause recirculation, and the presence of these local hydrodynamic eddies causes nonuniform convective mass transport, as shown in a number of studies of wet chemical etching, and in microelectronics [Occhialini and Higdon (1992); Kondo and Fukui (1998); Driesen et al. (2000); Georgiado et al. (2000)]. Flow in channel networks: has numerous applications; larger-scale examples include pollutant transport, predictive tools for modelling water supply resources, and surface waters, whereas small-scale examples include flow in capillary networks (human body, microfluidics), the cooling of electronic compounds, and chemical etching for the process of fabrication of electronic circuits. This paper focuses on the problem of flow in channel networks on a microscopic scale, with particular attention accorded to the conditions under which eddies arise in Stokes regime.

Recirculation motion in viscous flow is a problem of long-standing interest [Moffatt (1964), Taneda (1979), O'Neill (1983), Bourot (1984), Hellou and Coutanceau (1992), Pozrikidis (1992), Hajjam (1996), Wang (1996, 2002), Shankar et al.(2003)]. Most of these works concern individual channels or cavities, whereas the recirculation flow in a network of channels has received much less attention. Jeong (2001) analyzed the flow in a compartmented channel; comprising an infinite channel in which a semi-infinite wall is placed on its axis 
generating three semi-infinite channels. He studied the structure of the flow over a wide range of flow rates, showing the formation of recirculation motion in the channel of low flow rate, and illustrated how the fluid flows out by surrounding this motion.

In this study, we investigate the possible Stokes flow structures in a fluid domain formed by four orthogonal channels or flow branches. We present a complete study covering a variety of flow rate distributions, with particular analysis of cases which exhibited eddies.

\section{Fluid flow formulation}

The fluid flow domain indicated in figure 1a is formed by orthogonal flow channels of the same width $2 y_{0}$, connected by walls whose equation is expressed in a local frame by the following equation:

$$
\left(\frac{X_{L}}{a}\right)^{2 m}+\left(\frac{Y_{L}}{b}\right)^{2 m}=1
$$

where $X_{L}$ and $Y_{L}$ are the coordinates in the local frame indicated in figure 1. The parameters $a$ and $b$ represent the dimensions shown in figure 1 which permit to calculate the shape of the curve of connection given by equation (1). The values of $a$ and $b$ used are $a=b=4 y_{0}$. To avoid errors in calculation, the shape described by this equation contains no sharp corners. In our case, we chose $\mathrm{m}=4$, which leads to the shape described in figure 1 .

A viscous fluid of flow rates $q_{1}, \alpha\left|q_{1}\right|, \beta\left|q_{1}\right|$ and $\gamma\left|q_{1}\right|$ respectively, flows through the four branches; where $\alpha$, $\beta$ and $\gamma$ are dimensionless flow rates in branches 2,3 and 4 with respect to the real flow rate $\left|q_{1}\right|$ in branch 1 .

The sign of $\alpha, \beta$ and $\gamma$ must be consistent with the sign of $\vec{U}_{i} \cdot \vec{n}_{i}$, where $\vec{U}_{i}$ represents the mean normal velocity vector at the inlet of every branch, and the external normal is $\vec{n}_{i}$. Furthermore, the values $\alpha, \beta$ and $\gamma$ must respect the law of conservation as defined by the following equation:

$$
-1+\alpha+\beta+\gamma=0
$$

The fluid is assumed to be Newtonian and isothermal, with viscosity $\mu$ and density $\rho$ constant. Let $U_{i}$ be a characteristic value of the mean velocity in each branch. The local Reynolds number is defined as:

$$
R e_{i}=\frac{2 \rho U_{i} y_{0}}{\mu}
$$


1 It is assumed to be less than unity in order to meet the requirements for Stokes regime. More detailed 2 information about Stokes flow can be found in the following references: [Hasimoto and Sano (1980); Sangani and Acrivos (1982); Happel and Brenner (1986); Pozrikidis (1992); Hellou and Coutanceau (1992); Hajjam (1996); Higdon and Ford (1996); Wang (1996, 2002, 2009, 2010)].

Since it is assumed that the flow is both slow and two dimensional, we chose to solve the problem using the stream function. When inertia forces are negligible compared to viscous forces, the equation of two dimensional viscous flow known as Stokes equation is:

$$
\Delta \Delta \Psi=0
$$

where $\Psi$ is the stream function of the flow. In the case of a parallel-walled channel, the solutions to equation (4) are found respectively in the conditions of a plane Poiseuille flow (parabolic velocity profile), antisymmetric (zero mean rate flow and $\Psi(x, y)=\Psi(x,-y)$ ) and symmetric flow (zero mean rate flow and $\Psi(x, y)=-\Psi(x,-y))$. The corresponding stream functions $\left(\Psi_{P_{i}}, \Psi_{A_{i}}, \Psi_{S_{i}}\right)$ for each branch are given in appendix A; equations $\mathrm{A} 6, \mathrm{~A} 7$ and $\mathrm{A} 8$. The general solution is the combination of $\Psi_{P_{i}}, \Psi_{A_{i}}, \Psi_{S_{i}}$. Furthermore, a solution $\Psi_{0}$ is found for the flow in the junction of the flow branches; it is also given in appendix A; equation A9.

To determine the unknown coefficients of the stream function solutions $\left(\Psi_{0}, \Psi_{A_{i}}, \Psi_{S_{i}}\right)$ corresponding to each branch, and the stream function $\Psi_{0}$ in the junction, the following boundary conditions must be satisfied: for branches 1 and 3

$$
\begin{aligned}
& i=1, \quad i=3 \\
& -1 \leq x \leq 1
\end{aligned}
$$

$$
u_{0}(x, \pm 1)=v_{i}(0, x)
$$$$
v_{0}(x, \pm 1)=u_{i}(0, x)
$$$$
p_{0}(x, \pm 1)=p_{i}(0, x)
$$$$
\xi_{0}(x, \pm 1)=\xi_{i}(0, x)
$$
for branches 2 and 4

$$
\begin{gathered}
i=2, \quad i=4 \\
-1 \leq y \leq 1 \\
u_{0}( \pm 1, y)=u_{i}(0, y) \\
v_{0}( \pm 1, y)=v_{i}(0, y) \\
p_{0}( \pm 1, y)=p_{i}(0, y) \\
\xi_{0}( \pm 1, y)=\xi_{i}(0, y)
\end{gathered}
$$

where the velocity components $u$ and $v$, the pressure $p$ and the vorticity $\xi$ are calculated using the following relationhips:

$$
u=\frac{\partial \Psi}{\partial y}, v=-\frac{\partial \Psi}{\partial x}, \xi=-\Delta \Psi, \frac{\partial p}{\partial x}=\frac{\partial \xi}{\partial y}
$$


1 It is worth noting that the stream function $\Psi_{P_{i}}$ is entirely known when the flow rate in the corresponding

channel is done. These boundary conditions result, on the one hand, from the non-slip conditions on the walls of the flow branches, and on the other hand, from matching the flow branches to the flow junction. The matching conditions correspond to the continuity of the velocity vectors and the stress components, and it is easy to show that the continuity of the stress components leads to equality of pressure and vorticity. In addition to the conditions represented by equations (6), we must write the non-slip conditions on the curved parts of the boundaries (corners on figure 1) denoted by $c_{w i}$. These conditions are:

$$
u_{0}\left(x_{c w_{i}}, y_{c w_{i}}\right)=0, v_{0}\left(x_{c w_{i}}, y_{c w_{i}}\right)=0
$$

The quadratic minimisation method described in appendix B is applied to satisfy all the boundary conditions. The resulting equations are also developed in appendix B. The number of coefficients to be calculated is $N=N_{0}+\sum_{i=1}^{4}\left(N_{A_{i}}+N_{S_{i}}\right)$ where $N_{0}, N_{A_{i}}$ and $N_{S_{i}}$ are the number of terms retained for the functions $\Psi_{0}$, $\Psi_{A_{i}}$ and $\Psi_{S_{i}}$ respectively.

The width of the branches being equal, it was simple to take the same number of points on their interfaces with the central domain. Furthermore, the same number of terms in the series (A7) is considered for each branch. The system of equations resulting from quadratic minimization is solved using gauss elimination. The choice of $m_{i}=40, m_{0}=40, N_{A_{i}}=20$ and $N_{S_{i}}=20$ is considered to be satisfactory to calculate the motion with accuracy.

\section{Flow structures}

The conservation of mass and the hypothesis of two-dimensional Stokes flow lead to three independent configurations for the flow directions shown in figure 2. A parametric study is conducted by varying the flow rates in the channels. However, in order to limit the cases studied, particular flow rate distributions were analysed : $\alpha=\gamma$ and $\beta$ variable, or $\alpha=1$ and $\beta= \pm \gamma$ variable. Moreover, other original structures were selected.

\subsection{Configuration $1(\alpha=\gamma>0, \beta \geq 0)$}

For $\beta=0$, a flow of symmetrical cellular structure is established in branch 3 , as illustrated in figure 3.1. The fluid flows with open streamlines through branches 1, 2 and 4, however, the structure of the flow in branch 3 is radically different because of the occurrence of a recirculation flow as successive cells, symmetric to the axis, 
are bounded by separating streamlines, which are attached to the walls of this branch. By increasing $\beta$ slightly, the separating streamlines detach from the walls and the symmetrical cellular flow is consequently made up of only two recirculation cells, which are now located near the axis rather than being attached to the walls. The fluid flows out of branch 3 by circumventing these cells. The recirculation flow disappears when $\beta$ is greater than approximately 0.001 . In branch 3 , a structure was obtained in which all streamlines were open. Hence, the flow in the overall domain is composed of three flows, distinguished by two separation lines in the vicinity of the vertices of the junction, attached to the walls of branch 3; the separation lines are attached to these vertices for $\beta=1 / 3$. When $\beta$ approaches 0.98 (in this case $\alpha$ and $\gamma$ approach 0.01 ), two cells appear, each of which is attached to the lower wall of both branches 2 and 4 . This cell grows as $\beta$ increases. Finally for $\beta=1(\alpha=\gamma=0)$ we obtain purely recirculation flow in branches 2 and 4 . This flow is composed of a succession of antisymmetric eddies, whose properties were reported by Hellou and Coutanceau [1992].

In order to show other possible structures for configuration 1, we have selected two examples given in figure 3.2. Respectively, these examples show a case in which recirculation occurs in adjacent channels, and a case of dissymmetric structure $(\alpha \neq \beta \neq \gamma)$.

Flow reversal in branches 2 or 4 (inversion of the sign of $\alpha$ or $\gamma$ ) leads to configuration 2, as seen in figure 2 , while inversion of flow $\beta$ leads to configuration 3. However, simultaneous reversing of flows $\alpha$ and $\beta$ or $\beta$ and $\gamma$ or $\alpha$ and $\gamma$ results in structures conforming to configuration 1. Figure 3.3 shows examples of structures when $\alpha$ and $\gamma$ are reversed. It is worth noting that in this situation $\beta$ is necessarily greater than 1 . Thus, the structures obtained in figure 3.1 for $\beta$ between 0 and 1 , are now obtained for $\beta \in[1, \infty[$. For $\beta=1$, the structure in figure 3.1 remains unchanged when the flows $\alpha$ and $\gamma$ are reversed. Nevertheless, the structures obtained when $\beta$ approach zero exhibiting symmetric cells in branch number 3 (figure 3.1), can be obtained for flow reversal only for large values of $|\alpha|$ and $|\gamma|$ as shown in figure 3.3 where flow rates are 1000 times greater than in branch 1 . This example shows a structure closely similar to the structure shown in figure 3.1 corresponding to $\beta=0.0005$ but this situation would not be realistic under Stokes regime conditions. Finally, simultaneous inversion of the sign of $\alpha, \beta$ and $\gamma$ leads to an impossible situation under 2D conditions.

3.2. Configuration $2(\alpha>0, \gamma=-\beta, \beta \geq 0)$ 
The flow rate magnitude in branches 1 and 2 are assumed to be fixed at unity, and the flow rate magnitude $\beta$ and $\gamma$ vary in the range $[0,1]$. For $\beta=1$, the flow in the central field is divided into two equal, symmetrical parts either side of the straight line joining the opposite vertices common to branches 2-3 and branches 1-4 (figure 4.1). When $\beta$ decreases, this line maintains a curved shape and moves towards the branches with the lowest flow rate. Recirculation cells appear in the channels 2 and 3 for $\beta=0.02$ (or $\gamma=-0.02$ ). These cells grow in size as $\beta$ decreases, producing tortuous streamlines in the outflow. If $\beta$ is greater than unity, the structures obtained for $\beta \in[0,1]$ are now found for $\beta \in[1, \infty]$.

Figure 4.1 is completed by the structures given in figure 4.2 obtained when $\alpha=-\gamma$ and $\beta=1$. In this case, the recirculation flow is found to be developed in the opposite channels 2 and 4 for flow rate $\alpha$ less than 0.02 and the corresponding cells are attached to opposite walls.

\subsection{Configuration $3(\alpha>0, \gamma=-\beta, \beta \leq 0)$}

This configuration is of interest as it shows the structure for the flows in counter current. For $|\beta|=1$, the flow is divided into four equal parts, separated by the axes of the flow branches (see figure 5.1). The centre of the junction is a saddle point (i.e. a point of intersection of two streamlines). When $|\beta|$ decreases, the saddle point moves towards the common vertex between the branches of low flow rate. When $|\beta|$ is close to 0.1 , two separation streamlines become attached to the adjacent walls of the branches in which low flow occurs. Consequently, the communication between these branches is suppressed. For $|\beta|$ in the order of 0.02 , a cell attached to the other wall of each of these branches (walls connected respectively with branches 1 and 2 ) appears, this cell will grow as $|\beta|$ decreases. At the limit $(\beta=0)$, an antisymmetric sequence of eddies is formed.

As was the case for the previous configurations, we complete the illustrations with the cases presented in figure 5.2 corresponding to $\beta=-1$ and a ratio $\gamma / \alpha$ variable. Increasing this ratio leads to displacement of the saddle point along the axis of branches 2 and 4 towards the branch of small flow rate. In addition, we observe an original structure in the branch of small flow rate exhibiting two cells which each of them is attached separately to a wall of this branch. It is obvious that these cells will grow when $\gamma / \alpha$ more and more . A coalescing process would happen resulting in one cell of symmetric structure. 


\section{4. Conclusion}

3 We have conducted a study of the Stokes flow structures in flow branches linked by a junction, taking into consideration various flow rate distributions. The structures presented correspond to three possible configurations under 2D conditions, and particular streamline patterns exhibiting eddies were found. Such eddies develop within branches characterized by low or zero flow. For zero flow rate, these structures exhibit antisymmetric or symmetric closed streamlines, occupying the overall width of the channels in which they appear. For small flow rates, these eddies form original structures, occupying a part of the channel branches in which they develop, thus forcing the streamlines to surround them, creating positive pressure gradients. Although these eddy structures appear only in a limited range of flow rate, such structures may have consequences on transport processes. 


\section{References}

Adler, P. M. and H. Brenner (1984), Transport processes in spatially periodic capillary networks- Geometrical description and linear flow hydrodynamics, Pysico. Chem. Hydro., 5, 245-268.

Adler, P. M. and J.-F., Thovert, Fractures and fracture networks, Kluwer Acad., Norwell, Massachusets 1999.

Berkowitz, B., C. Naumann, and L. Smith (1994), Mass transfer at fracture intersections:An evaluation of mixing models, Water Resour. Res., 30(6), 1765-1773.

Bourot, J. M. (1984), Sur la structure cellulaire des écoulements plans de Stokes, à débit moyen nul, en canal indéfini à parois parallèles, C. R. Acad. Sci., 298 série II, 161-164.

Bourot, J. M. and F. Moreau (1987), Sur l'utilisation de la série cellulaire pour le calcul d'écoulements plans de Stokes en canal indéfini : Application au cas d'un cylindre circulaire en translation, Mech. Research Comm., 3, 187-197.

Bruderer, C. and Y. Bernabé (2001), Network modeling of dispersion: Transition from Taylor dispersion in homogeneous networks to mechanical dispersion in very heterogeneous ones, Water Resour. Res., 4, 897-908.

Bryden, M. D . and H. Brenner (1996), Multiple-timescale analysis of Taylor dispersion in converging and diverging flows, J. Fluid Mech., 311, 343-359.

Dentz, M. and B. Berkowitz (2003), Transport behavior of a passive solute in continuous time random walks and multirate mass transfer, Water Resour. Res., 39(5), WR001163.

Driesen, C. H., J. G. M. Kuerten and H. K. Kuiken (2000), Mass transport in a partially covered fluid-filled cavity, Int. J. Heat Mass Transfer, 43, 1823-1834.

Georgiadou, M., R. Mohr and R. C Alkire (2000), Local mass transport in two-dimensional cavities in laminar shear flow, $J$. Electrochem. Soc., 147, 3021-3028.

Hajjam, M., Contribution à l'étude de différents types d'écoulements en régime de Stokes, Thèse de Doctorat ès Sciences Physiques, Poitiers, France (in French), 1996.

Happel, J. and H. Brenner (1986), Low Reynolds number hydrodynamics. Mechanics of fluids and transport processes, Martinus Nijhof, Boston.

Hasimoto, H. and O. Sano (1980), Stokeslests and eddies in creeping flow, Ann. Rev. Fluid Mech., 12, 335-363.

Hellou, M. and M. Coutanceau (1992), Cellular Stokes flow induced by rotation of a cylinder in a closed channel, J. Fluid Mech., 236, 557-577.

Hellou, M. (2001), Sensitivity of cellular Stokes flow to geometry, Eur. J. Phys., 22, 67-77.

Higdon, J. J. L. and G. D. Ford (1996), Permeability of the three-dimensional models of fibrous porous media, J. Fluid Mech., 308, 341-361.

Jeong, J. T. (2001), Slow viscous flow in a partitioned channel, Phys. Fluids 13, 1577-1577 
.

8

9

30

Kondo, K. and K. Fukui (1998), Shape evolution of electrodeposited bumps with deep cavities, J. Electrochem. Soc., 145, 3007-3010.

Moffatt, H. K. (1964), Viscous and resistive eddies near a sharp corner. J. Fluid Mech. 18, 1-18.

Mourzenko, V. V., F. Yousefian, B. Kolbah, J.-F. Thovert, and P. M. Adler (2002), Solute transport at fracture intersections, Water Resour. Res., 1, 1-14.

Occhialini, J. M. and J. J. L. Higdon (1992), Convective mass transport from rectangular cavities in viscous flow, J. Electrochem. Soc., 139, 2845-2855.

O'Neill, M. E. (1983), On angles of separation in Stokes flow, J. Fluid Mech., 133, 427-442.

Park, Y. J. and K. K. Lee (1999), Analytical Solutions for Solute Transfer Characteristics at Continuous Fracture Junctions, Water Resour. Res., 35(5), 1531-1537.

Park, Y. J, K. K. Lee and B. Berkowitz (2001), Effects of junction transfer characteristics on transport in fracture networks, Water Resour. Res. 4, 909-923.

Park Y. J., K. K. Lee, G. Kosakowski and B. Berkowitz (2003), Transport behavior in three-dimensional fracture intersections, Water Resour. Res., 39 (8), 1215, doi:10.1029/2002WR001801, 2003.

Pozrikidis, C., Boundary integral and singularity methods for linearized viscous flow, Cambridge University Press, 1992.

Sangani, A. S. and A. Acrivos (1982a), Slow flow past periodic arrays of cylinders with application to heat transfer, Int. J. Multiphase Flow, 8, 193-206.

Shankar, P. N. , Kidambi, R., Hariharan, J. (2003), Oscillatory eddy structure in a container, J. Fluid Mech., 404, $163-185$.

Taneda, S. (1979), Visualizationof separating Stokes flow, J. Phys. Soc. Japan, 49, 1935-1942

Wang, C. Y. (1996), Stokes flow through an array of rectangular fibers, Int. J. Multiphase Flow, 1, 185-194.

Wang, C. Y. (2002), Slow viscous flow between hexagonal cylinders, Transport in porous media, 47, 67-80.

Wang, C. Y. (2009), The recirculating flow due to a moving lid on a cavity containing a Darcy -Brinkman medium, Applied Mathematical Modelling, 33, 2054-2061.

Wang, C. Y. (2010), Flow through a finned channel filled with a porous medium, Chemical Engineering Science, $65,1826-1831$. 
Appendix A: Resolution of the Stokes equation $\Delta \Delta \Psi=0$

\section{A1. General solution}

The separation of variables method is used to solve the following equations $\Delta \Psi_{1}=0$ and $\Delta \Psi=\Psi_{1}$. Thus, writing $\Psi_{1}=\Phi(x) \Theta(y)$ the following equations are obtained:

$$
\begin{aligned}
& \Phi^{\prime \prime}(x)-\sigma^{2} \Phi(x)=0 \\
& \Theta^{\prime \prime}(y)+\sigma^{2} \Theta(y)=0
\end{aligned}
$$

where the constant $\sigma$ may be either real or complex. The solution of this system leads to the following expressions respectively for $\sigma=0$ and $\sigma \neq 0$ :

$$
\begin{aligned}
& \Psi_{I_{0}}=A+B x+C y+D x y \\
& \Psi_{I_{\sigma}}=\sum_{\sigma}\left(A_{\sigma} e^{\sigma x}+B_{\sigma} e^{-\sigma x}\right)\left(C_{\sigma} e^{i \sigma y}+D_{\sigma} e^{-i \sigma y)}\right)
\end{aligned}
$$

where $A, B, C, D$ are real coefficients and $A_{\sigma}, B_{\sigma}, C_{\sigma}, D_{\sigma}$ are either real or complex coefficients. The function $\Psi_{l}=\Psi_{l_{0}}+\Psi_{l_{\sigma}}$ is harmonic, and consequently also biharmonic. We can easily show that the functions $x \Psi_{l}, y \Psi_{l},\left(x^{2}+y^{2}\right) \Psi_{1}$ are biharmonic. The sought stream function $\Psi$ which satisfies $\Delta \Delta \Psi=0$ is thus:

$$
\Psi=\Psi_{1}+x \Psi_{1}+y \Psi_{1}+\left(x^{2}+y^{2}\right) \Psi_{1}
$$

\section{A2. Solution in the case of a parallel-walled channel}

For a parallel-walled channel $i$, the stream function (A4) is written as:

$$
\Psi_{i}=\Psi_{P_{i}}+\Psi_{A_{i}}+\Psi_{S_{i}}
$$

which reveals, on the one hand, a flow with non-zero mean rate, represented by $\Psi_{P_{i}}$, and on the other hand, the combination of two flows with zero mean rate, represented by $\Psi_{A_{i}}$ and $\Psi_{S_{i}}$. The stream function $\Psi_{A_{i}}$ is antisymmetric $\left(\Psi_{A_{i}}\left(x_{i}, y_{i}\right)=\Psi_{A_{i}}\left(x_{i},-y_{i}\right)\right)$ while the stream function $\Psi_{S_{i}}$ is symmetric $\left(\Psi_{S_{i}}\left(x_{i}, y_{i}\right)=-\Psi_{S_{i}}\left(x_{i},-y_{i}\right)\right)$. The co-ordinates $\left(x_{i,} y_{i}\right)$ are dimensionless local co-ordinates $\left(x_{i}\right.$ along the axis of the channel $i$ ). 
To ensure non-slip wall conditions, we must suppress the terms factor of $x$ in equations (A2) and (A4). When the flow rate is non-zero, the combination of the equations A2 and A4 gives the following solution:

$$
\Psi_{P_{i}}=A_{i}-\frac{3}{4} q_{i}^{*}\left(\frac{y_{i}^{3}}{3}-y_{i}\right)
$$

which represents the dimensionless stream function of a flow of parabolic velocity profile (plane Poiseuille flow) corresponding to the dimensionless flow rate $q_{i}^{*} ; A_{i}$ is an arbitrary constant.

When the flow rate is zero, the expressions of $\Psi_{A_{i}}$ and $\Psi_{S_{i}}$ are determined by considering the combination $\Psi_{l_{\sigma}}+y \Psi_{l_{\sigma}}$. Writing $\sigma=\lambda+i \mu$ and after setting in real form, one finds:

$$
\begin{aligned}
& \Psi_{A i}\left(\operatorname{or} \Psi_{S i}\right)=\sum_{n} \exp \left(\lambda_{n} x_{i}\right)\left\{B_{n}\left(P_{n} \cos \mu_{n} x_{i}-Q_{n} \sin \mu_{n} x_{i}\right)\right. \\
& \left.-C_{n}\left(Q_{n} \cos \mu_{n} x_{i}+P_{n} \sin \mu_{n} x_{i}\right)\right\} \\
& D_{i}=1-y_{i}, S_{i}=1+y_{i} \\
& 2 P_{n}=S_{i} \sin \lambda_{n} D_{i} \cosh \mu_{n} D_{i} \pm D_{i} \sin \lambda_{n} S_{i} \cosh \mu_{n} S_{i}, \\
& 2 Q_{n}=S_{i} \cos \lambda_{n} D_{i} \sinh \mu_{n} D_{i} \pm D_{i} \cos \lambda_{n} S_{i} \sinh \mu_{n} S_{i}
\end{aligned}
$$

The upper and lower signs of (A7) relate to the functions $\Psi_{A_{i}}$ and $\Psi_{S_{i}}$ respectively.

The parameters $\lambda_{n}$ and $\mu_{n}$ are the eigenvalues of $\lambda$ and $\mu$ which satisfy the following equations:

$$
\begin{aligned}
& \sin 2 \lambda \cosh 2 \mu=\mp 2 \lambda, \\
& \cos 2 \lambda \sinh 2 \mu=\mp 2 \mu
\end{aligned}
$$

It is understood that equations (A7) are made non dimensional using $y_{0}$ and the absolute value of the flow rate $\left|q_{1}\right|$ as references.

The pairs $\left(-\lambda_{n}, \mu_{n}\right),\left(\lambda_{n},-\mu_{n}\right),\left(-\lambda_{n},-\mu_{n}\right)$ also satisfy equation (10). We consider the pair $\left(\lambda_{n}>0, \mu_{n}>0\right)$ for the negative semi-infinite field $-\infty<x_{i} \leq 0$ and $-1 \leq y_{i} \leq 1$. The pair $\left(-\lambda_{n},-\mu_{n}\right)$ is valid for the positive semi-infinite field. As coefficients $B_{n}$ and $C_{n}$ are arbitrary, the pairs $\left(-\lambda_{n}, \mu_{n}\right),\left(\lambda_{n},-\mu_{n}\right)$ do not change equation (A7). It is worth noting that the values of $\left(\lambda_{n}, \mu_{n}\right)$ were calculated up to $n=40$ with a high degree of accuracy [Bourot and Moreau, 1987].

Because equations (A8) are periodic, the stream functions $\Psi_{A_{i}}$ and $\Psi_{S_{i}}$ of each channel are zero on certain lines joining the walls of this branch. These lines are located periodically along this branch for each value of $n$. Thus, 
1 a flow with zero mean rate in a branch presents an infinite succession of lines known as separation lines 2 delimiting recirculation cells [O'Neill, 1983; Bourot, 1984; Hellou and Coutanceau, 1992; Hellou, 2001].

3 Previously, Moffatt [1964] showed that slow flow in a sharp corner presents this phenomenon when the angle is $4 \quad$ smaller than $146^{\circ} 3$.

5

\section{A3. Solution in the junction of the channels}

In the junction, called domain 0 , the flow rate is non-zero. In addition, it is assumed that no separation solutions can exist. Therefore, according to (A2) and (A4), the stream function representing the flow in this field is given by:

$$
\Psi_{0}=\sum_{m, p} A_{m, p} x^{m} y^{p}
$$

where $A_{m, p}$ are real coefficients, $m$ and $p$ are integers ranging from 0 to 3 . The pairs $(2,2),(2,3),(3,2)$ and $(3,3)$ are not permitted because they do not satisfy equation (A9).

\section{Appendix B: Quadratic minimisation method}

Let $g_{i}$ and $g_{0}$ represent the expressions of velocity components, pressure or vorticity in channels 1 to 4 and in the junction domain respectively. The expressions of cumulative quadratic errors committed on the matching conditions (equation (5)), and on the boundary conditions in the junction domain, equation (7), are respectively:

$$
S_{1}=\sum_{i=1}^{4} \sum_{m_{i}}\left(g_{0}-g_{i}\right)^{2}
$$

$$
S_{2}=\sum_{m_{0}} g_{0}^{2}
$$

where $m_{i}$ and $m_{0}$ respectively are the number of points on each interface and on the walls of the junction domain. The minimization of these errors is expressed by:

$$
\frac{\partial\left(S_{1}+S_{2}\right)}{\partial A_{j}}=0
$$




$$
\begin{aligned}
& g_{i}=q_{i}^{*} h_{i}\left(y_{i}\right)-\sum_{k=N_{0}+1}^{N_{i}} A_{k_{i}} f_{k_{i}}\left(x_{i}, y_{i}\right) \\
& i=1, \ldots, 4 \quad N_{i}=N_{0}+N_{A_{i}}+N_{S_{i}}
\end{aligned}
$$

$$
g_{0}=\sum_{k=1}^{N_{0}} A_{k 0} f_{k_{0}}(x, y)
$$

Of course in this setting, conveniently used to condense the expressions of velocity components, pressure and vorticity, the functions $f_{k_{0}}, h_{i}$ and $f_{k_{i}}$ are different according to which of the aforementioned expressions they represent. They are obtained without any particular difficulty using equations (A6), (A7) and the relationships (7). Applying minimization leads to writing the system of equations in the following condensed form:

$$
\sum_{m} \sum_{k=1}^{N} A_{k} f_{k} A_{j}=\sum_{i=1}^{4} q_{i}^{*} h_{i} f_{j}, j=1, \ldots, N
$$

where $m$ is the total number of minimization points. In this system, indices 0 and $i$, corresponding to the five domains, do not appear because of the classification of all the coefficients from 1 to $N$. In the calculation, this classification requires the cancellation on each interface of the functions concerning the other interfaces.

\section{Notation}

\begin{tabular}{ll}
\hline$a_{n}$ & complex roots of equations $\sin 2 a y_{0}= \pm 2 a y_{0}$ \\
$p$ & Pressure \\
$\vec{V}$ & velocity vector \\
$u$ & x-velocity component \\
$v$ & y-velocity component \\
$y_{0}$ & half width of a channel $i$ \\
$U_{i}$ & mean velocity in a channel $i$ \\
$q_{i}$ & flow rate in a channel numbered $i$ \\
$q_{i}^{*}=q_{i} / 2 U_{i} y_{0}$ & non dimensioned flow rate \\
& values of the flow rates in the channels relatively to the flow \\
$\alpha, \beta, \gamma$ & rate of reference ( e.g. $\left.q_{1}\right) \alpha=q_{2} / q_{1}, \beta=q_{3} / q_{1}, \gamma=q_{3} / q_{1}$ \\
$\lambda_{n}, \mu_{n}$ & Laplacian operator \\
$\xi$ & real and complex values of the roots $a_{n}$ \\
& stream function \\
& Vorticity
\end{tabular}




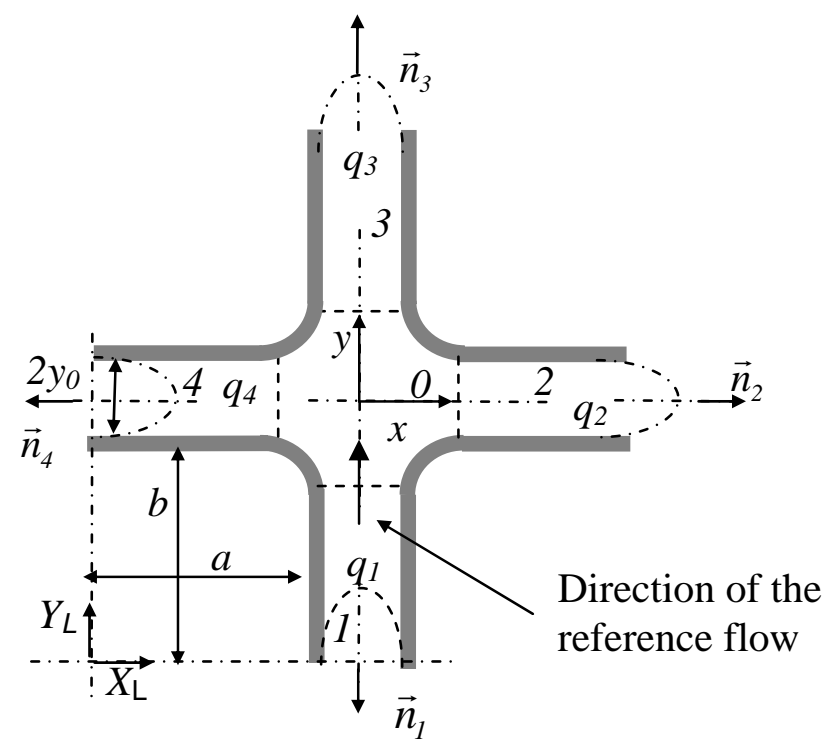

Figure 1. Delineation of the junction of four flow branches. The parabolic dashed lines represent the velocity profile at the inlet (far from the junction) if the flow rate is non null

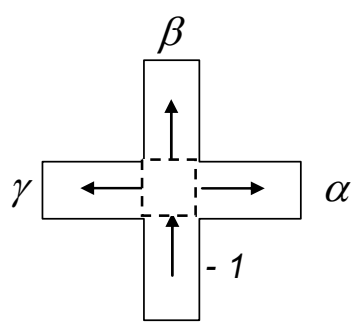

(1)

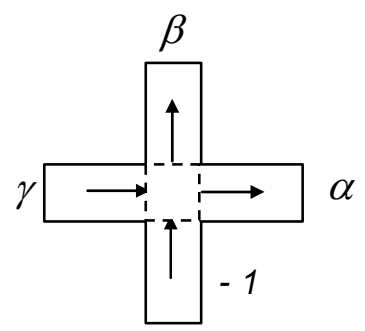

(2)

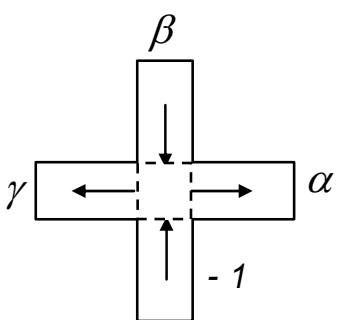

(3)

Figure 2. Schematisation of the flow directions for a two-dimensional junction of flow branches 

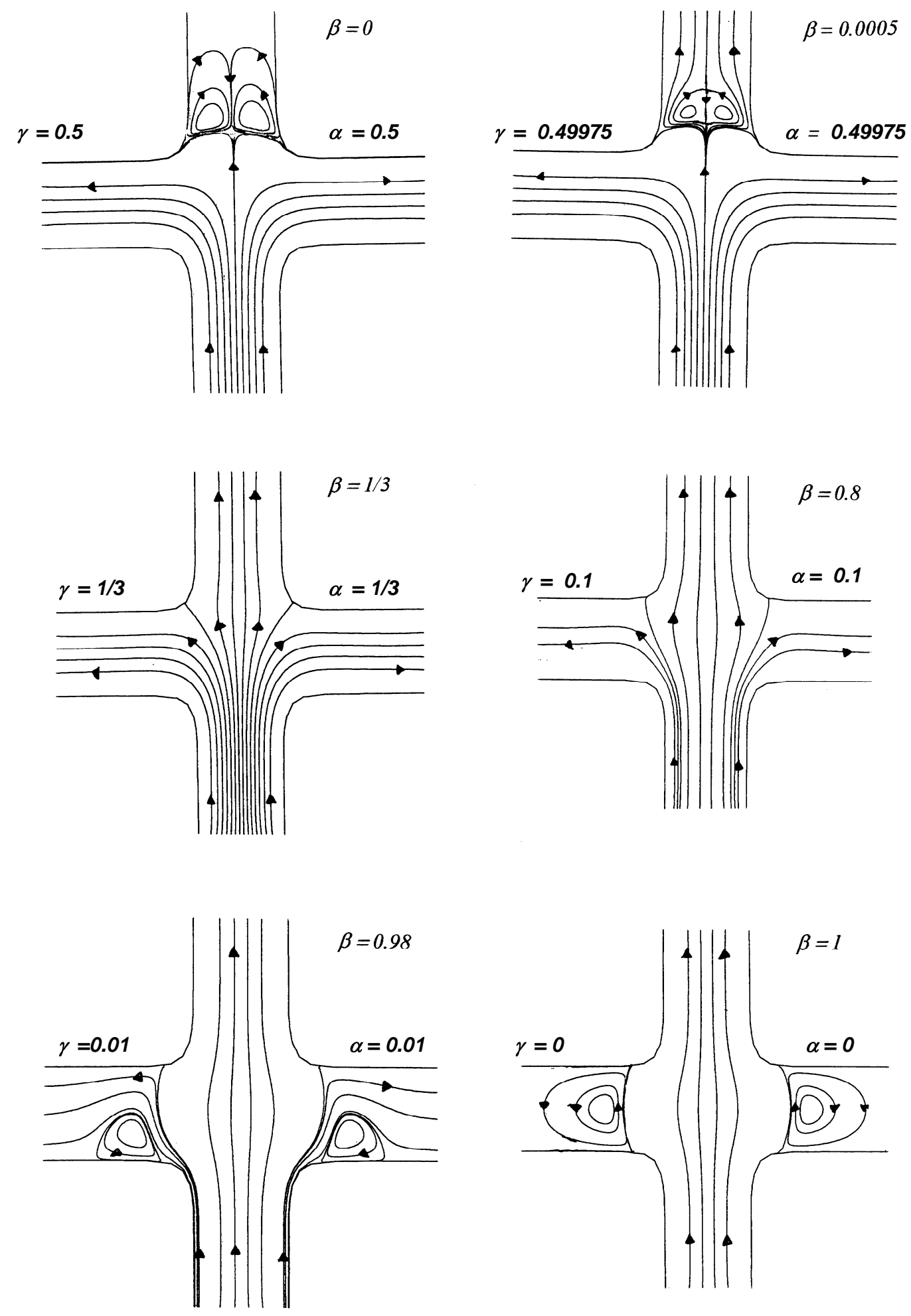

Figure 3.1 Streamline patterns for flow rates corresponding to configuration 1 under the following conditions : $-1, \alpha>0, \beta>0, \gamma=\alpha$. 

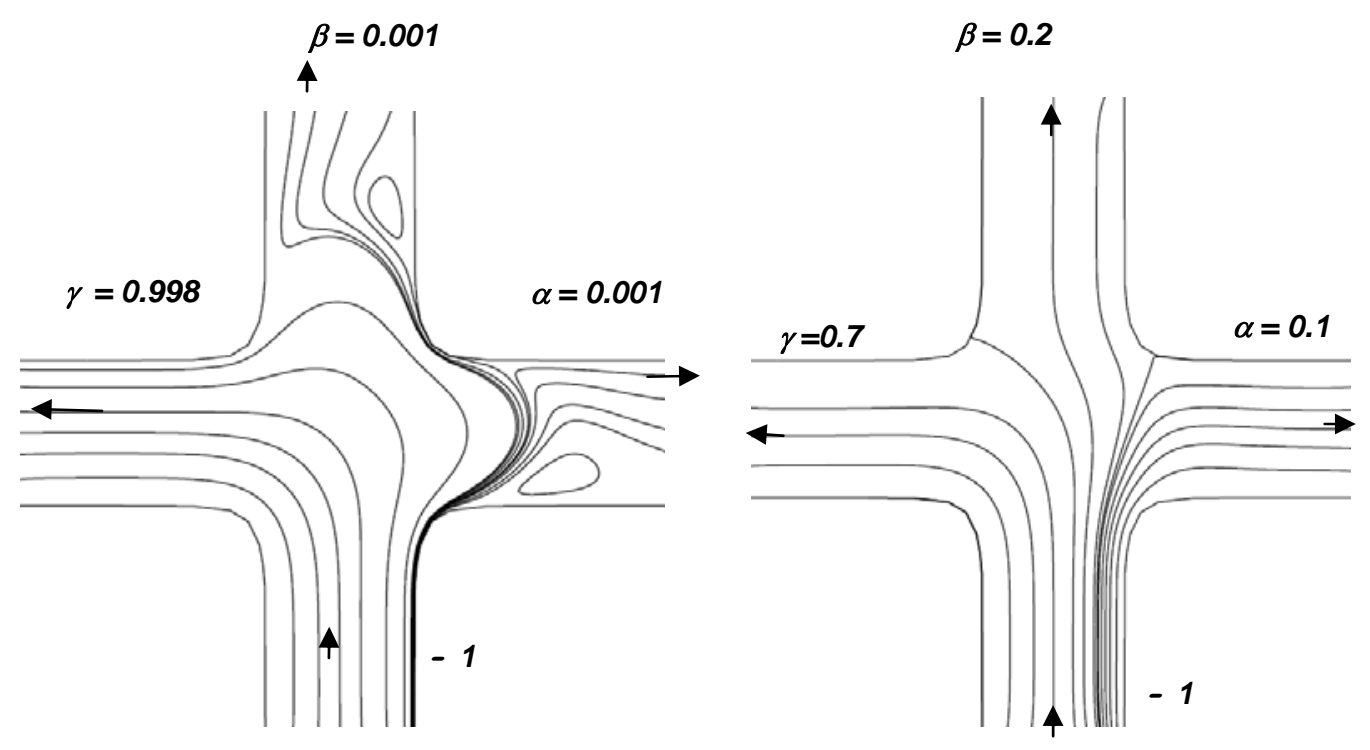

Figure 3.2 Streamline patterns for flow rates corresponding to configuration 1 under the following conditions : (a) $-1, \alpha>0, \alpha=\beta, \gamma>0$; (b) all the flow rates are different
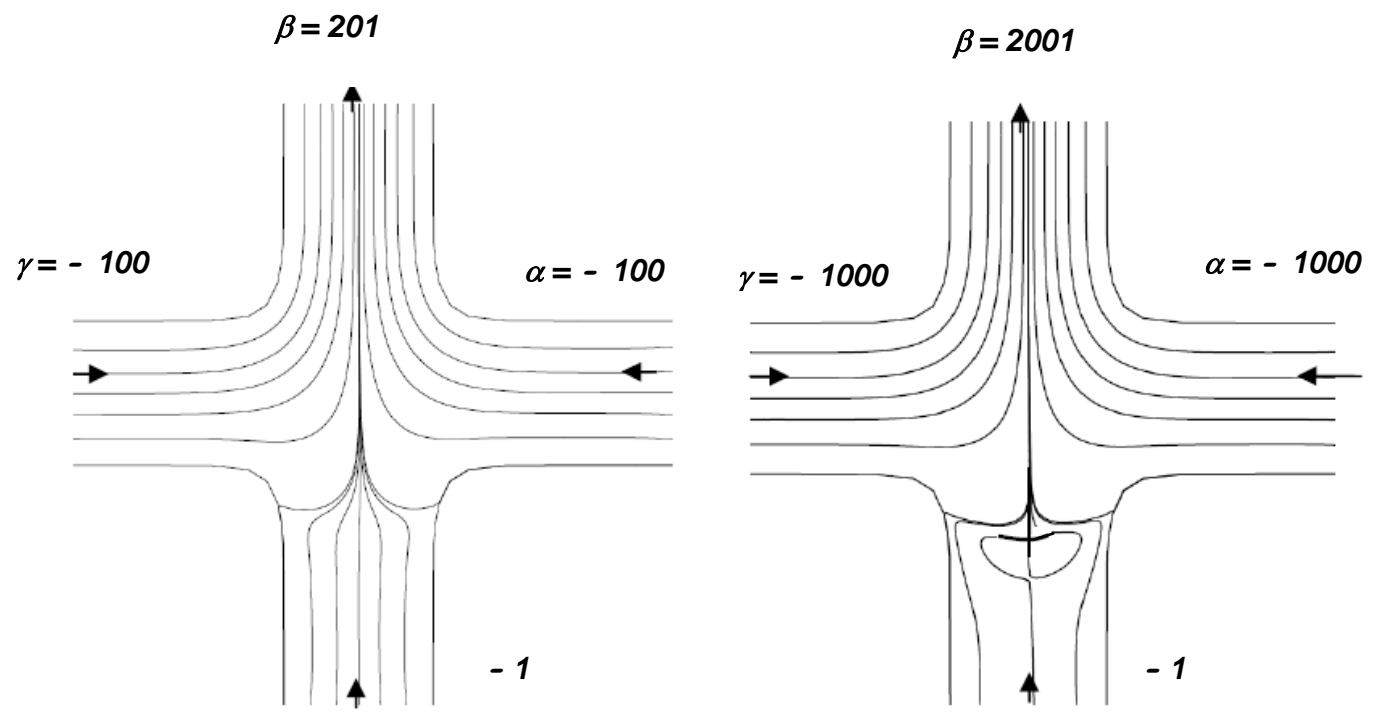

Figure 3.3 Streamline patterns for flow rates corresponding to configuration 1 reversed, under the following conditions : $-1, \alpha<0, \beta>1, \alpha=\gamma$ 

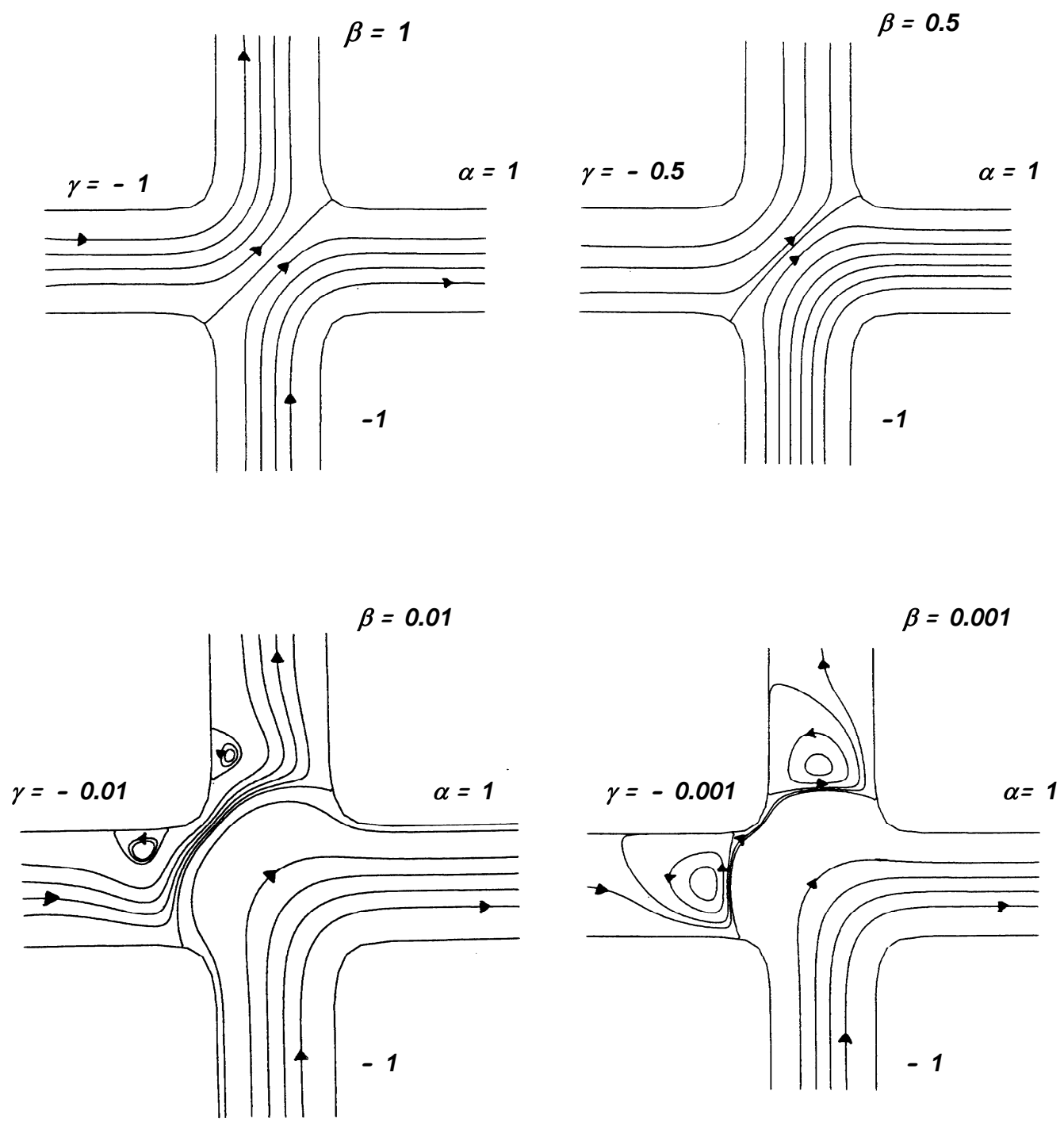

Figure 4.1. Streamline patterns for flow rates corresponding to configuration 2, under the following conditions : $-1, \alpha=1, \beta>0, \gamma=-\beta$. 

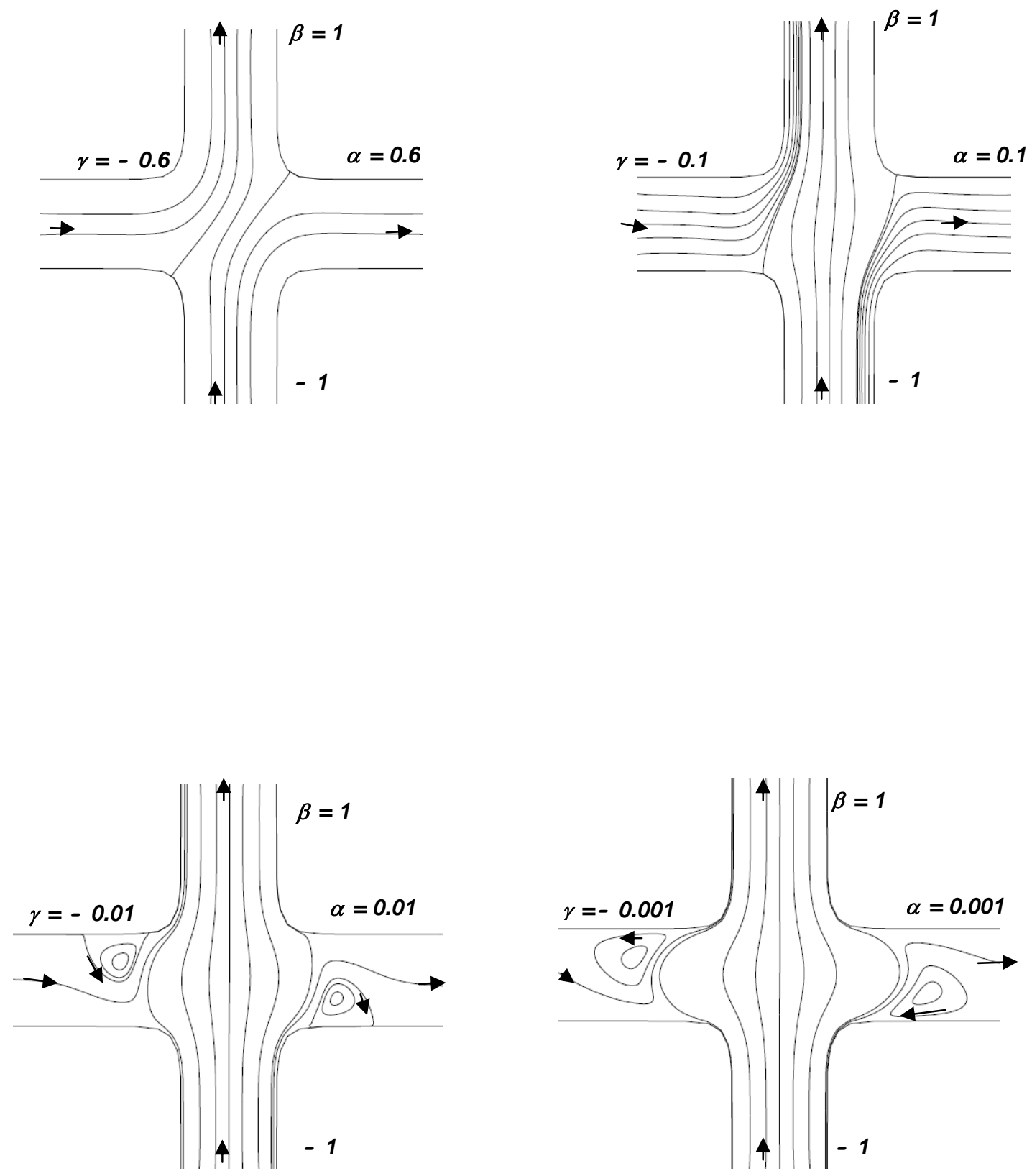

Figure 4.2 Streamline patterns for flow rates corresponding to configuration 2 under the following conditions : $-1, \alpha>0, \beta=1, \alpha=-\gamma$ 

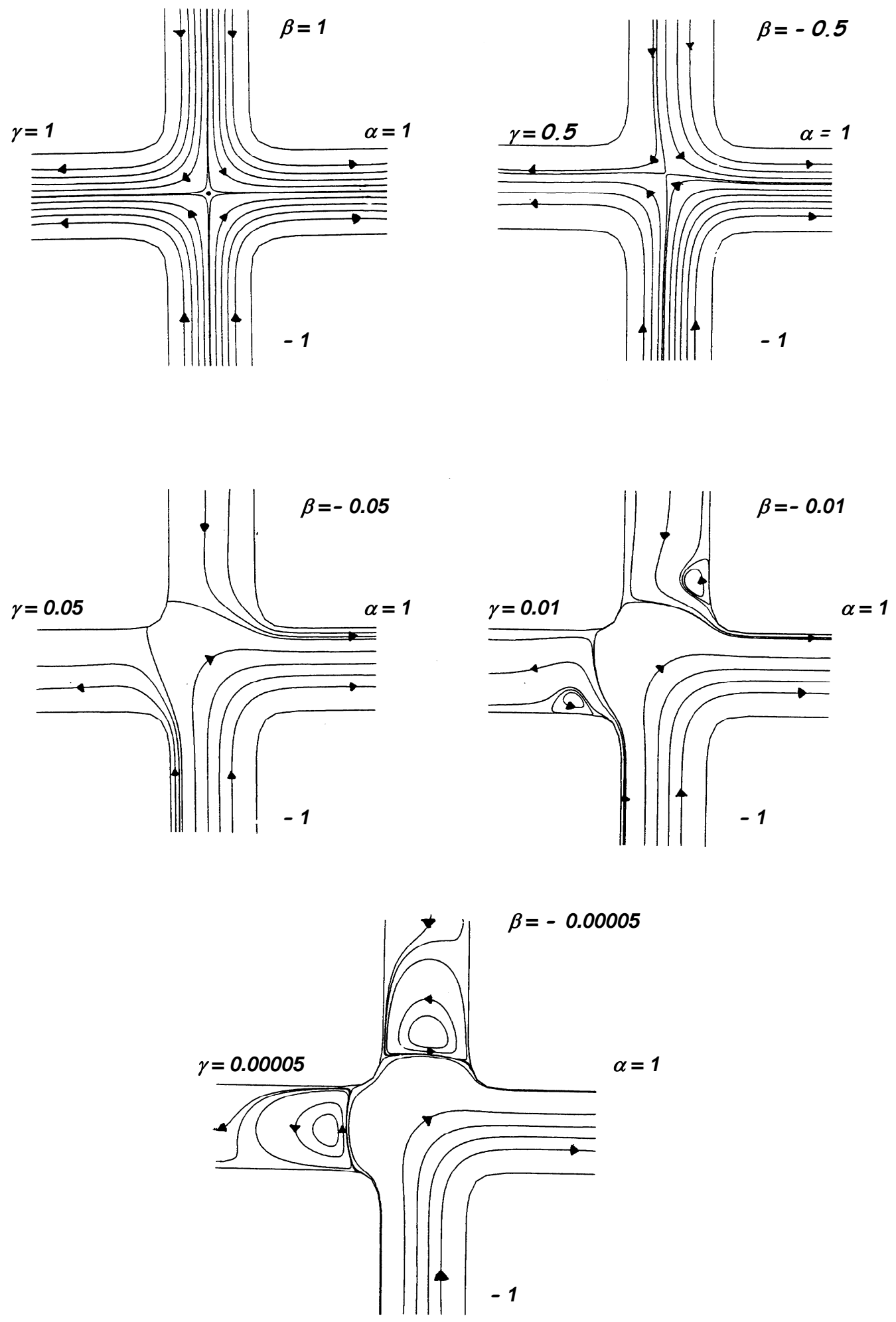

Figure 5.1. Streamline patterns for flow rates corresponding to configuration 1 under the following conditions : $\alpha=1, \beta<0, \gamma=-\beta$ 

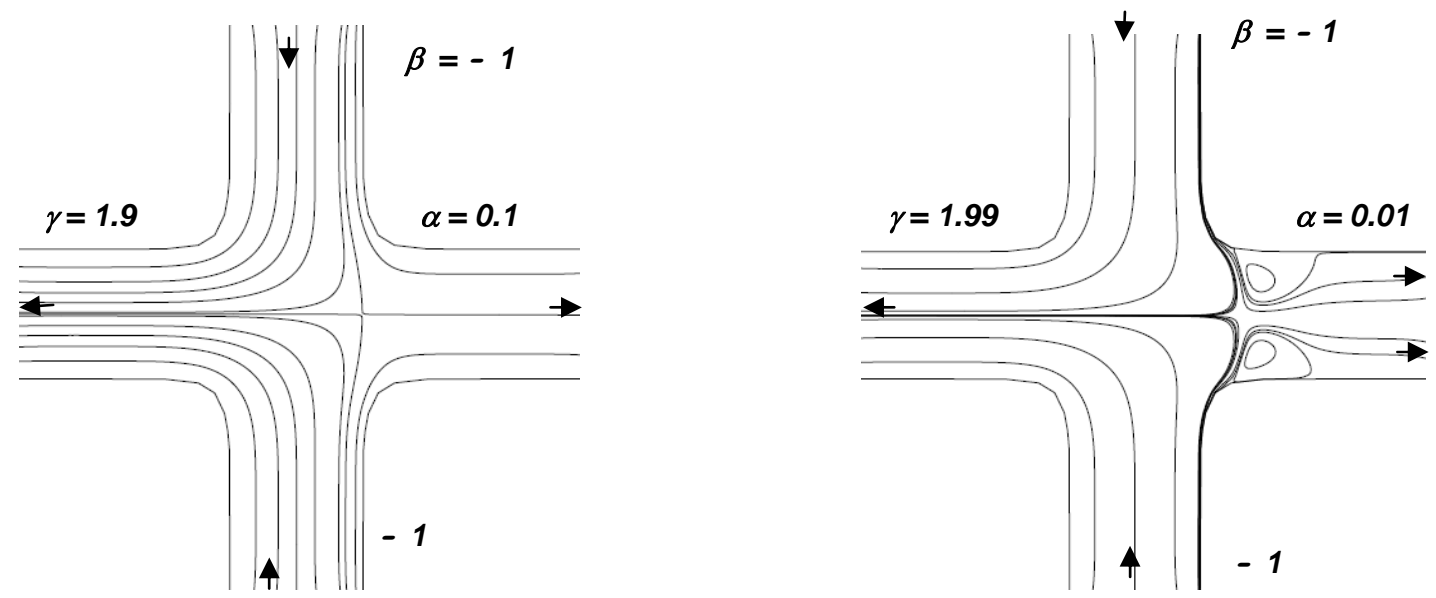

Figure 5.2. Two examples of streamline patterns when all the flow rates are different 